\title{
The real primes and the Riemann hypothesis Jamel Ghanouchi
}

RIME departmant of Mathematics

\section{Abstract}

In this document, we deal with the concept of real prime number together with the Riemann hypothesis to the real numbers. Thus, we highlight the new hypothesis by a calculus of integral.

\section{The approach}

The Rieman hypothesis states the non trivial zeros of the Riemann zeta function $\zeta(z)=\sum_{t=1}^{\infty} \frac{1}{t^{z}}$ lie on the critical line $\frac{1}{2}+i y$

\section{Definition}

A real number is composed if it can be written as

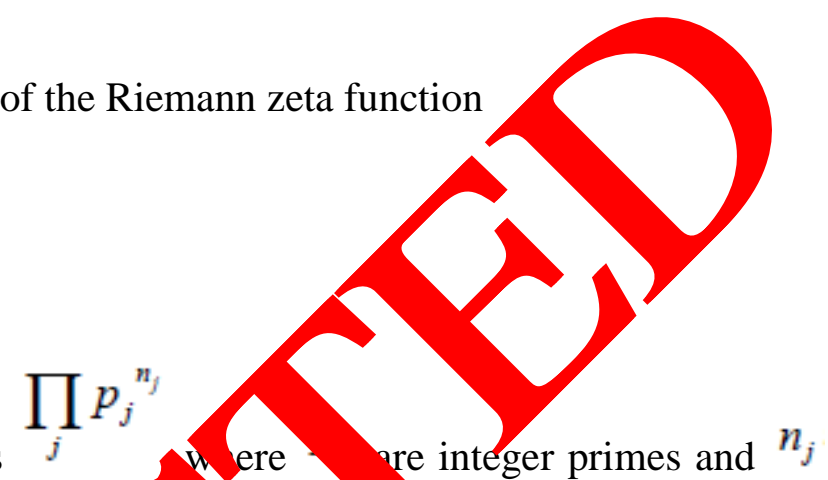
are rationals. A prime real number can be written only nubers like $\pi, \mathrm{e}, \ln (2)$. Thus $\sqrt[q]{p}=p^{\frac{1}{q}}$ is composed. $\sqrt[q]{p}+1 \neq p^{\frac{q}{q}}+1$ is prime and we have $\sqrt[2 t]{p}-1=(p-1)(\sqrt[2]{p}+1)^{-1}\left(\sqrt[2^{-1}]{p}+1\right)^{-1} \ldots\left(\sqrt{n_{n}}+1\right)^{-1}$ composed, for example.

For t integer, Euler has proved that $\zeta(z)=\sum_{t=1}^{\infty} \frac{1}{t^{z}}$

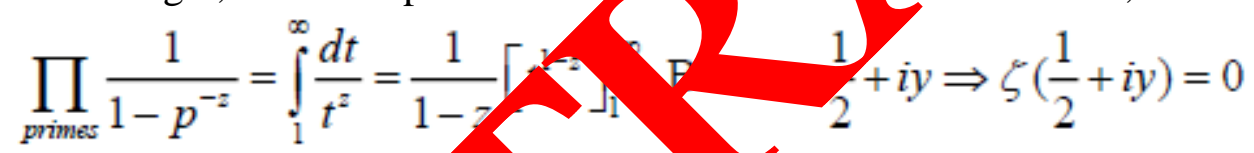

We have proved the hyp 4 for real abers. The Riemann hypothesis is important because it gives information abou the z of the Riemann function and the distribution of those zeros are related to real prim s?

\section{Conclusion}

We did esent $\mathrm{ke}$ this, but we have given a proof of the Riemann hypothesis for real

\section{Bibliograp}

[1] Karl Sabbagh, The Riemann hypothesis, the greatest unsolved problem in mathematics, Farrar, Straus and Giroux, 2004. 